Preprint-00

\title{
Energy transfer by the scattering of resonant photons
}

\author{
Avery Meiksin \\ SUPA $A^{\star}$, Institute for Astronomy, University of Edinburgh, Blackford Hill, Edinburgh EH9 $3 H J$, UK
}

14 October 2018

\begin{abstract}
A formal derivation is presented of the energy transfer rate between radiation and matter due to the scattering of an isotropic distribution of resonant photons. The derivation is developed in the context of the two-level atom in the absence of collisions and radiative transitions to and from the continuum, but includes the full angleaveraged redistribution function for photon scattering. The result is compared with previous derivations, all of which have been based on the Fokker-Planck approximation to the radiative transfer equation. A new Fokker-Planck approximation, including an extension to higher (post-diffusive) orders, is derived to solve the radiative transfer equation, and time-dependent numerical solutions are found. The relaxation of the colour temperature to the matter temperature is computed as the radiation field approaches statistical equilibrium through scattering. The results are discussed in the context of the Wouthuysen-Field mechanism for coupling the $21 \mathrm{~cm}$ spin temperature of neutral hydrogen to the kinetic temperature of the gas through $\operatorname{Ly} \alpha$ scattering. The evolution of the heating rate is also computed, and shown to diminish as the gas approaches statistical equilibrium.
\end{abstract}

Key words: atomic processess - cosmology: theory - line: formation - radiative transfer - radio lines: general - scattering

\section{INTRODUCTION}

The prospects for detecting the Intergalactic Medium (IGM) prior to reionization by extremely large radio telescopes like the Low Frequency Array (LOFAR) and the Square Kilometre Array (SKA) has led to a renewed interest the Wouthuysen-Field mechanism for coupling the spin temperature of neutral hydrogen to the kinetic temperature of the gas through the scattering of Ly $\alpha$ photons (Wouthuysen 1952; Field 1958). Following Field's (Field 1959a, b), analysis of the scattering of Ly $\alpha$ photons in a homogeneous medium and their role in setting the spin temperature of neutral hydrogen (Field 1958), the physics of $21 \mathrm{~cm}$ excitation and absorption was re-examined in the light of current cosmological models by Madau, Meiksin, \& Rees (1997, hereafter MMR) and Tozzi et al. (2000). A large number of various scenarios and effects have since been explored in the literature (see Bowman, Morales \& Hewett 2005 and references therein).

In addition to de-coupling the hydrogen spin temperature from the temperature of the Cosmic Microwave Background $(\mathrm{CMB}), \mathrm{MMR}$ showed that Ly $\alpha$ photons were in principle capable of heating cold hydrogen gas through recoils. The derivation was based on the momentum trans-

\footnotetext{
* Scottish Universities Physics Alliance
}

ferred to the hydrogen atoms by the Ly $\alpha$ photons after recoil (Field 1959b, Basko 1981). MMR supported their argument by identifying the recoil heating term in the radiative transfer equation for the scattering of the photons using a Fokker-Planck approximation with atomic recoil of Rybicki \& Dell'Antonio (1994), who extended previous Fokker-Planck treatments (Unno 1955; Harrington 1973; Basko 1981; Krolik 1990) to include the full Voigt line profile. While recognising that the energy exchange would cease once the radiation and matter reached thermal equilibrium, MMR did not discuss the role of photon diffusion through frequency on the energy exchange rate, as its effect on energy exchange is negligible for sufficiently cold gas. As the radiation field approaches statistical equilibrium through scatterings, however, the photon diffusion term reduces the net heating rate, which ultimately vanishes once the radiation field achieves statistical equilibrium at frequencies near resonance, with a colour temperature that matches the matter temperature. This will generally occur well before the radiation field reaches thermal equilibrium with the matter. The degree to which the approach to statistical equilibrium reduces the heating rate depends on the frequency range over which equilibrium is achieved. While it may be achieved in the core of the absorption profile on a timescale of the order of the mean free time for scattering at line-centre, it will take longer outside the core and significant heating may still persist. A central purpose of this paper is to derive the en-

(C) 0000 RAS 
ergy exchange rate from the full radiative transfer equation, justify the use of the Fokker-Planck equation for describing the exchange of energy between the radiation and the matter, and compute the evolution of the heating rate in this approximation for some simple applications. This is done in the approximation of the two-level atom, neglecting collisions and scatterings to and from the continuum. A second purpose it to compute the timescale over which the colour temperature relaxes to the kinetic temperature of the gas, as this is crucial for determining the signature of $21 \mathrm{~cm}$ emission or absorption through the Wouthuysen-Field mechanism.

Under the assumption that the Fokker-Planck equation of Rybicki \& Dell'Antonio (1994) adequately described the exchange of energy with the matter, Chen \& Miralda-Escudé (2004) applied Rybicki \& Dell'Antonio's cosmological version of the Fokker-Planck equation to the heating of the IGM, assuming that the comoving radiation density had reached a steady-state. In this case, the energy exchange between the radiation field and the matter virtually ceases, as in the static case, except for a residual amount arising as photons are redshifted through the resonance frequency. No estimate, however, is made of the time required to reach the equilibrium heating rate.

Following Deguchi \& Watson's (1985) formulation of the resonant photon scattering problem including stimulated emission, Rybicki (2006) presents an alternative derivation of the energy exchange rate using a Fokker-Planck approximation, and derives an approximate form for the radiative transfer equation that is structurally similar to the Kompaneets equation.

In order to solve the radiative transfer equation, a different Fokker-Planck approach is adopted here. The FokkerPlanck approximation derived is more complete than any previously formulated in this context. In particular, the formulation presented here explicitly conserves photon number and energy to second order in derivatives of the radiation field, as well as when extended to higher orders, permitting more accurate solutions to be obtained in a selfconsistent manner than was previously possible. This remedies a shortcoming of the Fokker-Planck treatment of Rybicki \& Dell'Antonio (1994), for which particle conservation was imposed by hand. Since then Rybicki (2006) ${ }^{\dagger}$ has presented an improved Fokker-Planck treatment. There are, however, some differences between the Fokker-Planck treatment provided here and Rybicki's. These are discussed below.

In the next section, the basic theory of resonance scattering is reviewed, including a derivation of the rate of energy exchange between the radiation field and the scattering medium. The Fokker-Planck approximation is discussed in $\S 3$, and applied to some simple problems in $\S 4$. A discussion of the results and conclusions are presented in $\S 5$.

$\dagger$ The present paper was substantially complete, but not submitted, prior to the posting of Rybicki (2006). Differences between the present work and Rybicki's are commented on in the text.

\section{BASIC THEORY}

\subsection{Radiative transfer and heating equations}

Statements of the radiative transfer equation in the literature are generally approximations, and not all are adequate for formulating the full energy transfer problem between the photons and the scattering medium. A standard form of the radiative transfer equation in terms of the photon energy density (Mihalas 1978, §2) in fact leads to the complete absence of energy transfer due to the implicit approximations made. In this section, a derivation of the radiative transfer equation satisfactory for treating the effects of energy exchange by the scatter of resonant photons is presented. A formal expression for the energy exchange rate for an isotropic radiation field is derived in this section which uses the full scattering integral, unlike all previous discussions which were based on the Fokker-Planck approximation.

In order to clarify the assumptions made in the radiative transfer equation for resonant photons, a derivation from basic principles is provided. The problem is formulated in a non-relativistic context. Polarisation is suppressed as well, but the results are readily generalised.

In a static medium, the rate of change of the energy in the radiation field at frequency $\nu$ at position $\mathbf{r}$ at time $t$ moving (in the laboratory frame) into direction $\hat{\mathbf{n}}$ per unit area per unit solid angle per unit time per unit frequency is (Mihalas 1978, Section 2.2)

$$
\begin{aligned}
\frac{D I_{\nu}(\mathbf{r}, t, \hat{\mathbf{n}})}{D t} & =\frac{\partial I_{\nu}(\mathbf{r}, t, \hat{\mathbf{n}})}{\partial t}+c \nabla I_{\nu}(\mathbf{r}, t, \hat{\mathbf{n}}) \\
& =c \eta_{\nu}(\mathbf{r}, \hat{\mathbf{n}}, t)-c \chi_{\nu}(\mathbf{r}, \hat{\mathbf{n}}, t) I_{\nu}(\mathbf{r}, t, \hat{\mathbf{n}}),
\end{aligned}
$$

where $I_{\nu}(\mathbf{r}, t, \hat{\mathbf{n}})$ is the specific intensity, $\eta_{\nu}$ is the specific emissivity (energy emitted per unit volume per unit solid angle per unit time per unit frequency), $\chi_{\nu}$ is the specific extinction (fraction of incident energy removed per unit length), and $c$ is the speed of light. (Finite light travel-time effects are neglected.)

For simplicity, it will be assumed that the emissivity and extinction both arise entirely due to scattering by a two-level atom. (Much of the following discussion is based on Mihalas 1978, §13-4. See also Milkey \& Mihalas 1973.) Scatterings to and from the continuum levels are neglected, as are excitations and ionizations due to collisions. Local isotropy of the radiation field is also assumed, although global variations with position are allowed. Under these assumptions, the rate of change of atoms in the upper state due to interactions involving the production of photons of energy $h \nu$ is given by

$$
\begin{aligned}
\frac{d n_{u}(\nu)}{d t} & =-n_{u}(\nu)\left(A_{u l}+B_{u l} J_{\nu}\right) \\
& +n_{l} B_{l u} \int_{0}^{\infty} d \nu^{\prime} R\left(\nu^{\prime}, \nu\right) J_{\nu^{\prime}}
\end{aligned}
$$

where $J_{\nu}=\oint(d \omega / 4 \pi) I_{\nu}$ is the usual angle-averaged intensity. ( $J_{\nu}=I_{\nu}$ for an isotropic radiation field.) The terms on the right hand side correspond, respectively, to spontaneous decays given by the Einstein coefficient $A_{u l}$, stimulated emissions described by the downward coefficient $B_{u l}$ and scatterings from the lower to upper level described by the upward coefficient $B_{l u}$. The scattering function $R\left(\nu^{\prime}, \nu\right)$ is the angle-averaged probability that a photon of frequency $\nu^{\prime}$ is scattered to frequency $\nu$ by an atom in the lower state. 
It is normalised by $\varphi(\nu)=\int_{0}^{\infty} d \nu^{\prime} R\left(\nu, \nu^{\prime}\right)$, where $\varphi(\nu)$ is the absorption profile of the atoms in the lower state. The function $n_{u}(\nu) d \nu$ describes the number density of atoms in the upper state capable of emitting photons in the frequency range $(\nu, \nu+d \nu)$ in the observer's frame. In general $n_{u}(\nu) / n_{u}$, where $n_{u}$ is the total number density of photons in the upper level, is not given by the natural emission profile $\psi(\nu)=\int_{0}^{\infty} d \nu^{\prime} R\left(\nu^{\prime}, \nu\right)$ and must be solved for.

The emissivity is then given by

$\eta_{\nu}=h \nu n_{u}(\nu) \frac{A_{u l}}{4 \pi}$

and the absorption coefficient by

$\chi_{\nu}=h \nu\left[n_{l} \frac{B_{l u}}{4 \pi} \phi_{\nu}-n_{u}(\nu) \frac{B_{u l}}{4 \pi}\right]$.

Expressed in terms of the radiation energy density $u_{\nu}=$ $4 \pi J_{\nu} / c$, the radiative transfer equation becomes

$$
\begin{aligned}
\frac{D u_{\nu}(\mathbf{r}, t)}{D t}= & \frac{\partial u_{\nu}(\mathbf{r}, t)}{\partial t}+c \nabla u_{\nu}(\mathbf{r}, t) \\
= & h \nu n_{u}(\nu) A_{u l}-h \nu n_{l} \frac{B_{l u}}{4 \pi} c \varphi(\nu) u_{\nu} \\
& \times\left[1-\frac{B_{u l}}{B_{l u}} \frac{n_{u}(\nu)}{n_{l} \varphi(\nu)}\right] .
\end{aligned}
$$

In most applications, the level populations will have reached equilibrium. Eq. (2) then gives

$n_{u}(\nu)=n_{l} \frac{B_{l u} \int d \nu^{\prime} R\left(\nu^{\prime}, \nu\right) u_{\nu^{\prime}}}{4 \pi A_{u l} / c+B_{u l} u_{\nu}}$.

Noting that the photon occupation number is $\mathcal{N}(\nu)=$ $c^{3} u_{\nu} /\left(8 \pi h \nu^{3}\right)$, the rate equation for $u_{\nu}$ may be expressed as

$$
\begin{aligned}
\frac{D u_{\nu}(\mathbf{r}, t)}{D t}= & \frac{\partial u_{\nu}(\mathbf{r}, t)}{\partial t}+c \nabla u_{\nu}(\mathbf{r}, t) \\
= & h \nu n_{l}(\mathbf{r}, t) c \frac{B_{l u}}{4 \pi} \frac{\int d \nu^{\prime} R\left(\nu^{\prime}, \nu\right) u_{\nu^{\prime}}(\mathbf{r}, t)}{1+\mathcal{N}(\nu, \mathbf{r}, t)} \\
& -h \nu n_{l}(\mathbf{r}, t) c \frac{B_{l u}}{4 \pi} \varphi(\nu) u_{\nu} \\
\times & \left\{1-\frac{\int d \nu^{\prime} R\left(\nu^{\prime}, \nu\right)\left(\frac{\nu^{\prime}}{\nu}\right)^{3} \mathcal{N}\left(\nu^{\prime}, \mathbf{r}, t\right)}{\varphi(\nu)[1+\mathcal{N}(\nu, \mathbf{r}, t)]}\right\},
\end{aligned}
$$

after using the relations between the Einstein coefficients. When the radiation reaches thermodynamic equilibrium, it becomes blackbody radiation at the same temperature as the matter, so that $D u_{\nu} / D t=0$. This permits a relation to be derived between $R\left(\nu, \nu^{\prime}\right)$ and $R\left(\nu^{\prime}, \nu\right)$ in detailed balance. Setting $\mathcal{N}(\nu)=1 /[\exp (h \nu / k T)-1]$ gives

$$
\frac{R\left(\nu^{\prime}, \nu\right)}{R\left(\nu, \nu^{\prime}\right)}=\left(\frac{\nu}{\nu^{\prime}}\right)^{3} \frac{e^{h \nu^{\prime} / k T}-1}{e^{h \nu / k T}-1} \rightarrow\left(\frac{\nu}{\nu^{\prime}}\right)^{3} e^{-h\left(\nu-\nu^{\prime}\right) / k T},
$$

where the limit applies for $h \nu>>k T$ and $h \nu^{\prime}>>k T$ corresponding to the classical limit of a dilute radiation field. This limiting form corresponds to the result of Deguchi \& Watson (1985) (noting the change in their normalization of $\left.R\left(\nu, \nu^{\prime}\right)\right)$. They claim their result is general, although it seems to apply only for low values of the photon occupancy.

Eq. (7) simplifies. Defining the coefficient $\chi_{0}(\mathbf{r}, t)=$ $n_{l}(\mathbf{r}, t)\left(B_{l u} / 4 \pi\right) h \nu_{0}$, where $\nu_{0}$ is the resonant frequency, af- ter simplification the equation of radiative transfer becomes

$$
\begin{aligned}
\frac{D u_{\nu}(\mathbf{r}, t)}{D t}= & \frac{\partial u_{\nu}(\mathbf{r}, t)}{\partial t}+c \nabla u_{\nu}(\mathbf{r}, t) \\
= & \frac{\chi_{0}(\mathbf{r}, t) c}{h \nu_{0}} h \nu\left[\int_{0}^{\infty} d \nu^{\prime} R\left(\nu^{\prime}, \nu\right) u_{\nu^{\prime}}\right. \\
& \left.-\varphi(\nu) u_{\nu}(\mathbf{r}, t)\right]+h \nu S(\nu) .
\end{aligned}
$$

A source term $S(\nu)$ of photons (per unit volume) has also been added (which may be taken to be an arbitrary function over space and time). It is noted that in the presence of additional interactions that are capable of redistributing the level populations, such as collisions or ionizations and recombinations to and from the continuum, the relations become considerably more involved. In general it is necessary to solve for the detailed level populations including the emission profile in order to establish the fraction of atoms in the lower and upper states. (See Mihalas 1978 §13-4 for details.)

An alternative form of the radiative transfer equation in terms of the number density of photons $n_{\nu}=u_{\nu} / h \nu$ is given by dividing Eq. (9) by $h \nu$

$$
\begin{aligned}
\frac{D n_{\nu}(\mathbf{r}, t)}{D t}= & \frac{\partial n_{\nu}(\mathbf{r}, t)}{\partial t}+c \nabla n_{\nu}(\mathbf{r}, t) \\
= & \frac{\chi_{0}(\mathbf{r}, t) c}{h \nu_{0}}\left[\int_{0}^{\infty} d \nu^{\prime} R\left(\nu^{\prime}, \nu\right) u_{\nu^{\prime}}\right. \\
& \left.-\varphi(\nu) u_{\nu}(\mathbf{r}, t)\right]+S(\nu) .
\end{aligned}
$$

The rate at which energy is transferred to the matter per unit volume is given by integrating Eq. (9) over frequency

$$
\begin{aligned}
G(\mathbf{r}, t)= & \int_{0}^{\infty} d \nu h \nu S(\nu)-\frac{D}{D t} \int_{0}^{\infty} d \nu u_{\nu}(\mathbf{r}, t) \\
= & -\frac{\chi_{0}(\mathbf{r}, t) c}{h \nu_{0}}\left[\int_{0}^{\infty} d \nu \int_{0}^{\infty} d \nu^{\prime} h \nu R\left(\nu^{\prime}, \nu\right) u_{\nu^{\prime}}(\mathbf{r}, t)\right. \\
& \left.\quad-\int_{0}^{\infty} d \nu h \nu \varphi(\nu) u_{\nu}(\mathbf{r}, t)\right] \\
= & \frac{\chi_{0}(\mathbf{r}, t) c}{h \nu_{0}} \int_{0}^{\infty} d \nu \int_{0}^{\infty} d \nu^{\prime}\left(h \nu^{\prime}-h \nu\right) R\left(\nu^{\prime}, \nu\right) u_{\nu^{\prime}}(\mathbf{r}, t)
\end{aligned}
$$

after noting that $\int d \nu R\left(\nu^{\prime}, \nu\right)=\varphi\left(\nu^{\prime}\right)$. The final form shows that the energy transfer rate to the scattering medium is given by the rate at which the photon energies in the observer's frame shift during scattering.

Eqs. (9) and (11) have some noteworthy symmetry properties that may be used to gain insight into the origin of the energy exchange in special situations. For resonant scattering, the redistribution function $R\left(\nu^{\prime}, \nu\right)$ for most applications is well-approximated as a function of $\left(\nu-\nu^{\prime}\right)$. Similarly $\varphi(\nu)$ is an (even) function of $\left(\nu-\nu_{0}\right)$. For a source function $S_{\nu}$ that is a function of $\left(\nu-\nu_{0}\right)$, in the absence of an initial radiation field, the form of Eq. (9) shows that the radiation energy density $u_{\nu}$ may also be expressed as a function $u\left(\nu-\nu_{0}\right)$. It is then possible to express $R\left(\nu, \nu^{\prime}\right)$ and $u\left(\nu-\nu_{0}\right)$ as sums of their even and odd parts in $\left(\nu-\nu_{0}\right), R\left(\nu, \nu^{\prime}\right)=R^{(e)}\left(\nu, \nu^{\prime}\right)+R^{(o)}\left(\nu, \nu^{\prime}\right)$ and $u\left(\nu-\nu_{0}\right)=u^{(e)}\left(\nu-\nu_{0}\right)+u^{(o)}\left(\nu-\nu_{0}\right)$, where $R^{(e)}\left(\nu, \nu^{\prime}\right)=$ $\left[R\left(\nu, \nu^{\prime}\right)+R\left(\nu^{\prime}, \nu\right)\right] / 2, R^{(o)}\left(\nu, \nu^{\prime}\right)=\left[R\left(\nu, \nu^{\prime}\right)-R\left(\nu^{\prime}, \nu\right)\right] / 2$, 
$u^{(e)}\left(\nu-\nu_{0}\right)=\left[u\left(\nu-\nu_{0}\right)+u\left(\nu_{0}-\nu\right)\right] / 2$ and $u^{(o)}\left(\nu-\nu_{0}\right)=$ $\left[u\left(\nu-\nu_{0}\right)-u\left(\nu_{0}-\nu\right)\right] / 2$. The form of Eq. (11) then shows that energy exchange between the radiation field and the matter is driven only by the even-odd combinations $R^{(e)} u^{(o)}$ and $R^{(o)} u^{(e)}$. In particular, if the source function $S_{\nu}$ is an even function of $\left(\nu-\nu_{0}\right)$, then $u\left(\nu-\nu_{0}\right)$ will have an odd part only if $R\left(\nu, \nu^{\prime}\right)$ does. If instead $R\left(\nu, \nu^{\prime}\right)=R\left(\nu^{\prime}, \nu\right)$, as holds for the redistribution Cases I, II and III (Mihalas 1978, §133) without atomic recoil $\ddagger$, then $u\left(\nu-\nu_{0}\right)$ will be even in $\left(\nu-\nu_{0}\right)$ and no energy exchange will occur. It is evident from Eq. (8) that the asymmetry induced by atomic recoil gives rise to the energy exchange in this case. These symmetry relations are exploited below.

It is shown in the Appendix that under the assumption of Case IIr redistribution (perfectly sharp lower state, finite radiative decay time in upper state, frequency coherence in the restframe of the atom), which is the case of most interest here, the integral over the redistribution function may be expressed in terms of the Voigt line profile $\varphi_{V}(\nu)$, so that

$$
\begin{aligned}
G(\mathbf{r}, t)= & \frac{\chi_{0}(\mathbf{r}, t) c}{h \nu_{0}} h \int_{0}^{\infty} d \nu u_{\nu}(\mathbf{r}, t)\left[\epsilon \omega \varphi_{V}(\nu)\right. \\
& \left.-\frac{1}{2} \omega^{2} \frac{d \varphi_{V}(\nu)}{d \nu}\right],
\end{aligned}
$$

where the effects of atomic recoil have been included to order $\epsilon=h \nu_{0} /\left(2 k T m_{a} c^{2}\right)^{1 / 2}$, where $T$ is the gas temperature and $m_{a}$ is the mass of the recoiling atom, and $\omega=\nu_{0} b / c$, where $b=\left(2 k T / m_{a}\right)^{1 / 2}$ is the Doppler parameter. Because $\varphi_{V}(\nu)$ is an even function of $\left(\nu-\nu_{0}\right)$, Eq. (12) shows that energy transfer by recoil (the first term in brackets) is driven by the even part of $u_{\nu}=u\left(\nu-\nu_{0}\right)$, while energy transfer through the second term is driven by the odd part. The odd part may also contain a term of order $\epsilon$, as it will for an even source.

It is shown below that in the Fokker-Planck approximation, a source function $S_{\nu}$ even in $\left(\nu-\nu_{0}\right)$ (or one wellapproximated as even, such as a function very smoothly varying across the resonance frequency), will generate an odd part to $u\left(\nu-\nu_{0}\right)$ only through recoils. Thus both terms in Eq. (12) will be proportional to the recoil parameter $\epsilon$. It is also noted that any non-negligible variation in frequency of the continuum source across the resonant frequency may induce non-negligible energy transfer between the radiation and matter even in the absence of recoils. Expanding $S_{\nu} \approx S_{\nu_{0}}+\left(\nu-\nu_{0}\right)(d S / d \nu)_{0}$ shows that the correction term $\left(\nu-\nu_{0}\right)(d S / d \nu)_{0}$ will drive an odd contribution to $u_{\nu}=u\left(\nu-\nu_{0}\right)$ in Eq. (9) even for a symmetric redistribution function $\left(R\left[\nu, \nu^{\prime}\right]=R\left[\nu^{\prime}, \nu\right]\right)$, and so create a non-vanishing energy exchange rate as described by Eq. (11).

Following Rybicki (2006), it is useful to recast Eq. (12) in terms of the radiation colour temperature. Motivated by Field's (1958) introduction of an effective colour temperature of the radiation field, MMR presented a formal definition of colour temperature $T_{\mathcal{N}}$ (called $T_{\alpha}$ in their paper) in terms of the photon occupation number $\mathcal{N}(\nu)$ :

$\ddagger$ Technically, redistribution Cases I, II and III do not allow for atomic recoils. The modifications with recoils shall be designated by adding the suffix 'r', thus, Case IIr.
$T_{\mathcal{N}}=-\frac{h}{k}\left[\frac{d \log \mathcal{N}(\nu)}{d \nu}\right]^{-1}$.

In order to account for stimulated scattering, Rybicki (2006) modified the temperature definition to $T_{\mathcal{N}}^{*}(\nu)=T_{\mathcal{N}}(\nu)[1+$ $\mathcal{N}(\nu)]$.

In light of the form of Eq. (12), it is now suggested that, in the absence of stimulated emission, the most natural definition for the colour temperature for describing energy exchange is in terms of the energy density of the radiation field. Through straightforward manipulations, Eq. (12) may be recast in the form

$G=P_{l} n_{l} \frac{h \nu_{0}}{m_{a} c^{2}} h \nu_{0}\left(1-\frac{T}{\left\langle T_{u}\right\rangle_{H}}\right)$,

where

$P_{l}=\frac{B_{l u}}{4 \pi} c \int_{0}^{\infty} d \nu \varphi_{V}(\nu) u_{\nu}$

is the total photon scattering rate per atom in the lower state, and the mean harmonic colour temperature has been defined by:

$\left\langle T_{u}\right\rangle_{H}=\int_{0}^{\infty} d \nu u_{\nu} \varphi_{V}(\nu) / \int_{0}^{\infty} d \nu u_{\nu} \varphi_{V}(\nu) \frac{1}{T_{u}(\nu)}$,

where

$T_{u}(\nu)=-\frac{h}{k}\left(\frac{d \log u_{\nu}}{d \nu}\right)^{-1}$.

The limit $T<<\left\langle T_{u}\right\rangle_{H}$ of Eq. (14) recovers the heating rate given by MMR. At lower colour temperatures, the heating rate is suppressed by the efficiency factor $1-T /\left\langle T_{u}\right\rangle_{H}$.

The temperature in Eq. (16) is identical to the "light temperature" $T_{L}$ defined by Field (1958) in the context of the Wouthuysen-Field mechanism, as will now be demonstrated. If the total transition rates induced by the scattering of Ly $\alpha$ photons from the lower hydrogen hyperfine $n=1$ level $\left({ }_{0} S_{1 / 2}\right)$ in hydrogen to the upper hyperfine level $\left({ }_{1} S_{1 / 2}\right)$ is designated $P_{01}^{L}$, and the reverse rate designated $P_{10}^{L}$, then Field (1958) (see also Tozzi et al. 2000), shows that in equilibrium,

$\frac{P_{01}^{L}}{P_{10}^{L}}=3 \frac{\left\langle u_{\nu_{0}}\right\rangle+\left\langle u_{\nu_{0}^{\prime}}\right\rangle}{\left\langle u_{\nu_{1}}\right\rangle+\left\langle u_{\nu_{1}^{\prime}}\right\rangle}$,

where $\left\langle u_{\nu_{i}}\right\rangle=\int d \nu \varphi_{V}\left(\nu-\nu_{i}\right) u_{\nu}$ is the energy density of the radiation field averaged over a Voigt profile centred on $\nu=\nu_{i}$. The four frequencies $\nu_{0}, \nu_{0}^{\prime}, \nu_{1}$ and $\nu_{1}^{\prime}$ correspond to the Ly $\alpha$ transitions between the $n=1$ and $n=2$ pairs $\left({ }_{0} S_{1 / 2,{ }_{1}} P_{1 / 2}\right),\left({ }_{0} S_{1 / 2,1} P_{3 / 2}\right),\left({ }_{1} S_{1 / 2,1} P_{1 / 2}\right)$, and $\left({ }_{1} S_{1 / 2},{ }_{1} P_{3 / 2}\right)$, respectively. Field (1958) defines the effective colour temperature $T_{L}$ of the radiation field by $P_{01}^{L} / P_{10}^{L}=$ $3\left(1-T_{*} / T_{L}\right)$, where $k T_{*}=h \nu_{01}$ and $h \nu_{01}$ is the energy difference between the $n=1$ hyperfine levels. A straightforward expansion of the Voigt profiles about $\varphi_{V}\left(\nu-\nu_{0}\right)$ to lowest order in $d \varphi_{V} / d \nu$ shows that $T_{L}$ is identical to $\left\langle T_{u}\right\rangle_{H}$, further justifying this as the most pragmatic definition of the colour temperature in the absence of stimulated emission (cf Deguchi \& Watson 1985).

Rybicki (2006) expresses the heating rate in a form similar to Eq. (14), except in terms of $T_{\mathcal{N}}^{*}(\nu)$ (his eq. [32]), instead of $T_{u}(\nu)$. He then approximates the scattering cross- 
section as a $\delta$-function to evaluate the colour temperature at line-centre to obtain (his eq. [35]):

$G_{\mathrm{Ryb}}=P_{l}^{*} n_{l} \frac{h \nu_{0}}{m_{a} c^{2}} h \nu_{0}\left[1-\frac{T}{T_{\mathcal{N}}^{*}\left(\nu_{0}\right)}\right]$.

(In Rybicki's formulation, $u_{\nu}$ is approximated as $h \nu_{0} n_{\nu}$ in the frequency-integral in the definition of $P_{l}$, and stimulated emission is accounted for by multiplying the result by $[1+\mathcal{N}(\nu)]$, the combined effect of which is defined here as $P_{l}{ }^{*}$.) The derivation here shows that the result is more general, and in particular does not rely on the Fokker-Planck formulation. It does, however, depend on the specific form Eq. (12).

The various definitions of colour temperature have relative differences from $T_{u}$ of $k T_{u} / h \nu$. Provided $k T_{u}<<h \nu$, the differences in these definitions are therefore small. Moreover, because multiplicative factors of $\nu$ were dropped in adopting the Voigt profile (so, for example, the Rayleigh scattering limit is not recovered), the actual multiplicative factors of $\nu$ in defining the radiation colour temperature are not accounted for in this formalism. For these reasons, the approximation $u_{\nu}=h \nu_{0} n_{\nu}$ is adopted below for the definition of the colour temperature for convenience, to give $T_{n}(\nu)$ in analogy to Eq. (17):

$\left\langle T_{n}\right\rangle_{H}=\int_{0}^{\infty} d \nu n_{\nu} \varphi_{V}(\nu) / \int_{0}^{\infty} d \nu n_{\nu} \varphi_{V}(\nu) \frac{1}{T_{n}(\nu)}$

where

$T_{n}(\nu)=-\frac{h}{k}\left(\frac{d \log n_{\nu}}{d \nu}\right)^{-1}$

\subsection{Uniform expansion}

In a homogeneous and isotropic expanding medium, Eq. (9) must be modified by adding expansion and redshifting terms to $\partial u_{\nu} / \partial t$. For this case, $D u_{\nu} / D t$ becomes (Peebles 1968; Peebles 1993),

$\frac{D u_{\nu}}{d t}=\frac{\partial u_{\nu}}{\partial t}+3 \frac{\dot{R}}{R} u_{\nu}-\nu \frac{\dot{R}}{R} \frac{\partial u_{\nu}}{\partial \nu}$,

where $R(t)$ is the expansion factor. The equation may be simplified further by defining $\tilde{u}_{\nu}=\left(R / R_{0}\right)^{3} u_{\nu}$ and $\tilde{S}_{\nu}=$ $\left(R / R_{0}\right)^{3} S_{\nu}$, where $R_{0}$ is the expansion factor at some time $t_{0}$. In this case, the form of Eq. (9) is recovered if $u_{\nu}$ and $S_{\nu}$ are everywhere replaced by $\tilde{u}_{\nu}$ and $\tilde{S}_{\nu}$, and $D \tilde{u}_{\nu} / D t$ is now identified with

$\frac{D \tilde{u}_{\nu}}{D t}=\frac{\partial \tilde{u}_{\nu}}{\partial t}-\nu \frac{\dot{R}}{R} \frac{\partial \tilde{u}_{\nu}}{\partial \nu}$.

A similar result follows for the number density $n_{\nu}$ by first expressing $u_{\nu}=h \nu n_{\nu}$ in Eq. (10), and then replacing $n_{\nu}$ everwhere by $\tilde{n}_{\nu}=\left(R / R_{0}\right)^{2} n_{\nu}, S_{\nu}$ by $\tilde{S}_{\nu}=\left(R / R_{0}\right)^{2} S_{\nu}$, and identifying $D n_{\nu} / D t$ with

$\frac{D \tilde{n}_{\nu}}{D t}=\frac{\partial \tilde{n}_{\nu}}{\partial t}-\nu \frac{\dot{R}}{R} \frac{\partial \tilde{n}_{\nu}}{\partial \nu}$.

An integration of the rate equation for $u_{\nu}$ exactly recovers the heating rate of Eq. (11), after expressing $D u_{\nu} / D t$ by Eq. (22), which allows for the energy lost by the radiation due to the expansion.

\subsection{Approximate formulations}

Several approximate form of the transfer equation for resonant radiation exist in the literature. A common form starts from Eq. (9), but sets $h \nu=h \nu_{0}$ in the coefficient, ignoring the frequency shift of a photon on scattering (Mihalas 1978, $\S 2-1)$. The equation for the energy density $u_{\nu}$ then becomes

$$
\frac{D u_{\nu}(\mathbf{r}, t)}{D t} \cong \chi_{0}(\mathbf{r}, t) c\left[\int_{0}^{\infty} d \nu^{\prime} R\left(\nu^{\prime}, \nu\right) u_{\nu^{\prime}}(\mathbf{r}, t)-\varphi(\nu) u_{\nu}(\mathbf{r}, t)\right]
$$

While adequate for describing the evolution of $u_{\nu}$ for most cases, this approximate form is inadequate for the purpose of computing the rate of energy transfer between the photons and the scattering medium, since the rate should be proportional to the frequency shift per scattering event. A straightforward integration over frequency, noting that $\int d \nu R\left(\nu^{\prime}, \nu\right)=\varphi\left(\nu^{\prime}\right)$, shows that this form for the scattering equation gives a vanishing value for the net energy exchange rate.

An intermediate approximation between the exact form and the approximation above is provided by Basko (1978) for the photon number density $n_{\nu}$. This starts from Eq. (10), but makes the approximations $u_{\nu^{\prime}}=h \nu_{0} n_{\nu^{\prime}}$ and $u_{\nu}=h \nu_{0} n_{\nu}$ on the right hand side to arrive at

$\frac{D n_{\nu}(\mathbf{r}, t)}{D t} \cong \chi_{0}(\mathbf{r}, t) c\left[\int_{0}^{\infty} d \nu^{\prime} R\left(\nu^{\prime}, \nu\right) n_{\nu^{\prime}}(\mathbf{r}, t)-\varphi(\nu) n_{\nu}(\mathbf{r}, t)\right]$

Most Fokker-Planck treatments in the literature start with this form. It has the advantage over Eq. (25) of recovering an approximate form for the heating rate, given by multiplying by $h \nu$ and integrating over frequency

$$
\begin{aligned}
G(\mathbf{r}, t) & =\int_{0}^{\infty} d \nu S(\nu)-\frac{D}{D t} \int_{0}^{\infty} d \nu u_{\nu}(\mathbf{r}, t) \\
& \cong \chi_{0}(\mathbf{r}, t) c \int_{0}^{\infty} d \nu \int_{0}^{\infty} d \nu^{\prime}\left(h \nu^{\prime}-h \nu\right) R\left(\nu^{\prime}, \nu\right) n_{\nu^{\prime}}(\mathbf{r}, t) .
\end{aligned}
$$

It is identical to Eq. (11) except that $u_{\nu^{\prime}}$ has been approximated by $h \nu_{0} n_{\nu^{\prime}}$. Since the expression already allows for a frequency shift of the scattered photon, however, this approximation introduces only a small, and normally negligible, error.

\section{FOKKER-PLANCK APPROXIMATION}

\subsection{Basic equation}

The integro-differential character of the radiative transfer equation with the full redistribution function makes its solution nearly intractable, requiring special numerical methods. A useful technique is to obtain solutions in a FokkerPlanck approximation. Basko (1978) introduced a FokkerPlanck approximation for Eq. (26) for an infinite homogeneous medium, although he included only the dominant contribution to the line profile in the wings. Krolik (1990) presents a formal expression for the Fokker-Planck approximation, but similarly evaluates the coefficients only in the wings. Rybicki \& Dell'Antonio generalised Basko's form by rederiving a Fokker-Planck equation retaining the full Voigt line profile. Following Unno (1955) and Harrington (1973), they expand $n_{\nu^{\prime}}$ in Eq. (26) in a Taylor series about $\nu=\nu^{\prime}$. 
Their derived coefficients, however, did not explicitly conserve photon number, and so they were forced to adjust the coefficients to ensure the number of photons is conserved. An alternative derivation was presented by Rybicki (2006) invoking detailed balance and stimulated emission. It is still, however, based on a Taylor series expansion of $n_{\nu}$.

A derivation of the Fokker-Planck equation is presented here which explicitly conserves both particle number and energy while still retaining the full line profile. For the present formulation, it is useful to introduce the probability $W(\nu, Q)$ for scattering a photon of frequency $\nu$ to one of frequency $\nu^{\prime}=\nu-Q$. Eq. (9) may then be re-expressed as

$$
\begin{aligned}
\frac{D u_{\nu}(\mathbf{r}, t)}{D t}= & \frac{\chi_{0}(\mathbf{r}, t) c}{h \nu_{0}} h \nu\left[\int_{-\infty}^{\infty} d Q^{\prime} W\left(\nu^{\prime}, Q^{\prime}\right) u_{\nu^{\prime}}\right. \\
& \left.-\int_{-\infty}^{\infty} d Q W(\nu, Q) u_{\nu}\right]+h \nu S(\nu),
\end{aligned}
$$

where $Q^{\prime}=\nu^{\prime}-\nu$, and $\varphi(\nu)=\int d Q W(\nu, Q)$ has been used. It is also assumed here and elsewhere that $W(\nu, Q)$ becomes vanishingly small for large $Q$, so that the lower integration bound $\nu^{\prime}=0$ in the first integral may be well-approximated by $Q^{\prime} \rightarrow-\infty$. Unlike previous approximations, the standard Fokker-Planck approximation is based on a Taylor series expansion of the product of both the scattering probability function and the distribution function. A Taylor series expansion of $W\left(\nu^{\prime}, Q^{\prime}\right) u_{\nu^{\prime}}$ about $\nu^{\prime}=\nu$ gives, to second order in $\nu^{\prime}-\nu$,

$$
\begin{aligned}
W\left(\nu^{\prime}, Q^{\prime}\right) u_{\nu^{\prime}} \approx & W\left(\nu, Q^{\prime}\right) u_{\nu}+Q^{\prime} \frac{\partial}{\partial \nu}\left[W\left(\nu, Q^{\prime}\right) u_{\nu}\right] \\
+ & \frac{1}{2} Q^{\prime 2} \frac{\partial^{2}}{\partial \nu^{2}}\left[W\left(\nu, Q^{\prime}\right) u_{\nu}\right] .
\end{aligned}
$$

Substituting this in Eq. (28) gives the canonical FokkerPlanck form

$$
\begin{aligned}
\frac{D u_{\nu}(\mathbf{r}, t)}{D t} \cong & \frac{\chi_{0}(\mathbf{r}, t) c}{h \nu_{0}} h \nu \frac{\partial}{\partial \nu}\left\{\langle Q\rangle \varphi(\nu) u_{\nu}(\mathbf{r}, t)\right. \\
& \left.+\frac{1}{2} \frac{\partial}{\partial \nu}\left[\left\langle Q^{2}\right\rangle \varphi(\nu) u_{\nu}(\mathbf{r}, t)\right]\right\}+h \nu S(\nu),
\end{aligned}
$$

where

$\left\langle Q^{n}\right\rangle \varphi(\nu) \equiv\left[\int_{-\infty}^{\infty} d Q Q^{n} W(\nu, Q)\right]$,

and the dummy integration variable $Q^{\prime}$ has been replaced by $Q$. This form conserves energy to the accuracy of the approximation and to order $b / c$, as may be shown by an integration by parts over $\nu$ and noting that $W(\nu, Q)$ vanishes at the integration boundaries,

$$
\begin{aligned}
G & =\int_{0}^{\infty} d \nu h \nu S(\nu)-\frac{D}{D t} \int_{0}^{\infty} d \nu u_{\nu}(\mathbf{r}, t) \\
& =\frac{\chi_{0}(\mathbf{r}, t) c}{h \nu_{0}} \int_{0}^{\infty} d \nu h\langle Q\rangle \varphi(\nu) u_{\nu}(\mathbf{r}, t) \\
& =\frac{\chi_{0}(\mathbf{r}, t) c}{h \nu_{0}} \int_{0}^{\infty} d \nu \int_{0}^{\infty} d \nu^{\prime}\left(h \nu-h \nu^{\prime}\right) R\left(\nu, \nu^{\prime}\right) u_{\nu}(\mathbf{r}, t)
\end{aligned}
$$

identical to Eq. (11). This justifies estimating the energy exchange rate between the photons and the gas using the Fokker-Planck approximation, provided the approximation yields an accurate solution for the radiation field.
Dividing Eq. (30) through by $h \nu$ gives the FokkerPlanck equation for the photon number density

$$
\begin{aligned}
\frac{D n_{\nu}(\mathbf{r}, t)}{D t}= & \frac{\chi_{0}(\mathbf{r}, t) c}{h \nu_{0}} \frac{\partial}{\partial \nu}\left\{\langle Q\rangle \varphi(\nu) u_{\nu}\right. \\
& \left.+\frac{1}{2} \frac{\partial}{\partial \nu}\left[\left\langle Q^{2}\right\rangle \varphi(\nu) u_{\nu}\right]\right\}+S(\nu),
\end{aligned}
$$

which is in explicit photon number conservation form (cf Krolik 1990). The coefficients $\langle Q\rangle$ and $\left\langle Q^{2}\right\rangle$ are evaluated in the Appendix for Case IIr redistribution. For $\epsilon=0$, they are identical to those derived by Rybicki \& Dell'Antonio (1994) (noting that they expand in $-Q$ in the notation here). They did not explicitly include the recoil terms, instead adding by hand the result from Basko (1981). Here the recoil terms are included self-consistently, resulting in additional higher order corrections.

It is convenient to solve the equation in non-dimensional form. Defining the dimensionless frequency shift $x=(\nu-$ $\left.\nu_{0}\right) / \omega$ and the conformal time $\tau=\int_{0}^{t} d t \chi_{0}(\mathbf{r}, t) c / \omega$, and substituting in the moments of $Q$ in terms of the dimensionless Voigt profile $\phi_{V}(x)=\varphi_{V}(\nu) \omega$, Eq. (33) becomes

$$
\begin{aligned}
\frac{D n_{x}(\mathbf{r}, \tau)}{D \tau}- & \frac{D \log \omega}{D \tau} n_{x}(\mathbf{r}, \tau) \\
= & \frac{\partial}{\partial x}\left\{\left[\epsilon \phi_{V}(x)-\frac{1}{2} \frac{d \phi_{V}(x)}{d x}\right] n_{x}(\mathbf{r}, t)\right\} \\
& +\frac{1}{2} \frac{\partial^{2}}{\partial x^{2}}\left\{\left[\phi_{V}(x)-\frac{4}{3} \epsilon \frac{d \phi_{V}(x)}{d x}\right.\right. \\
& \left.\left.+\frac{1}{3} \frac{d \phi_{V}^{2}(x)}{d x^{2}}\right] n_{x}(\mathbf{r}, t)\right\}+\tilde{S}(x) \\
= & \frac{1}{2} \frac{\partial}{\partial x}\left[2 \epsilon \phi_{V}(x) n_{x}(\mathbf{r}, t)+\phi_{V}(x) \frac{\partial}{\partial x} n_{x}(\mathbf{r}, t)\right] \\
& +\frac{1}{6} \frac{\partial^{2}}{\partial x^{2}}\left\{\left[-4 \epsilon \frac{d \phi_{V}(x)}{d x}+\frac{d^{2} \phi_{V}(x)}{d x^{2}}\right] n_{x}(\mathbf{r}, t)\right\} \\
+ & \tilde{S}(x) \\
= & \frac{1}{2} \frac{\partial}{\partial x}\left[2 \epsilon \phi_{V}(x) n_{x}(\mathbf{r}, t)+\phi_{V}(x) \frac{\partial}{\partial x} n_{x}(\mathbf{r}, t)\right. \\
& \left.+\frac{2}{3} \epsilon \frac{d^{2} \phi_{V}(x)}{d x^{2}} n_{x}(\mathbf{r}, t)+\frac{1}{3} \frac{d^{2} \phi_{V}(x)}{d x^{2}} \frac{\partial}{\partial x} n_{x}(\mathbf{r}, t)\right] \\
+ & \frac{1}{6} \frac{\partial}{\partial x}\left\{\left[-6 \epsilon \frac{d^{2} \phi_{V}(x)}{d x^{2}}+\frac{d^{3} \phi_{V}(x)}{d x^{3}}\right] n_{x}(\mathbf{r}, t)\right. \\
& \left.-4 \epsilon \frac{d \phi_{V}(x)}{d x} \frac{\partial}{\partial x} n_{x}(\mathbf{r}, t)\right\}+\tilde{S}(x),
\end{aligned}
$$

where $n_{x}(\mathbf{r}, \tau)=n_{\nu}(\mathbf{r}, t) \omega$ and $\tilde{S}(x)=S(\nu) \omega^{2} /\left(\chi_{0} c\right)$ have been defined, a possible time-evolution of $\omega$ has been allowed for, and terms of order $x b / c$ have been neglected, consistent with the adoption of the Voigt profile (see the Appendix). It should be noted that the valid recovery of the effects of atomic recoil in this approximation requires $b / c<<2 \epsilon$. For hydrogen Ly $\alpha$, this corresponds to $T<<h \nu_{0} / k \approx 10^{5} \mathrm{~K}$.

The approximations made by Rybicki \& Dell'Antonio (1994) correspond to excluding the term in curly braces in the second equality of Eq. (34). The result has the form of a diffusion equation with diffusion coefficient $\phi_{V}(x) / 2$. The same form results from the full Fokker-Planck equation when higher order derivatives of $\phi_{V}(x)$ are neglected, as is 
appropriate far in the wings of the profile (Harrington 1973; Basko 1978, 1981). It will be referred to here as the FokkerPlanck diffusion approximation (FPDA), to distinguish it from the standard Fokker-Planck approximation (FPA).

The formulation of Rybicki (2006) (his eq. [20]), corresponds to excluding the term in curly braces in the third equality of Eq. (34). It is another FPDA equation. It is adopted here with the diffusion coefficient $D(x)=\phi_{V}(x)+$ $(1 / 3) d^{2} \phi_{V}(x) / d x^{2}$ (his eq. [11]). The equation also includes an extra term of order $b / c$ (arising from the term $-2 n_{\nu} / \nu$ in his eq. [20]), and a quadratic term in $n_{x}$ arising from stimulated emission. These latter two terms, not included in the discussion here, play pivotal roles when the gas temperature approaches the equivalent temperature of the resonant frequency or as the radiation thermalises with the matter, which, in the case of an optically thick medium, results in blackbody radiation. Comparisons with the approximation of Rybicki (2006) are confined here to the diffusion equation terms using the diffusion coefficient above.

\subsection{Higher order extension}

The above formalism is readily extended to higher orders. This is useful when solving the equations to test the degree of convergence of the original second-order Fokker-Planck approximation. Extended to fourth order (by continuing the expansion in Eq. (29) to fourth order), Eq. (34) takes the form

$$
\begin{aligned}
& \frac{D n_{x}(\mathbf{r}, \tau)}{D \tau}-\frac{D \log \omega}{D \tau} n_{x}(\mathbf{r}, \tau) \\
= & \frac{\partial}{\partial x}\left\{\left[\epsilon \phi_{V}(x)-\frac{1}{2} \frac{d \phi_{V}(x)}{d x}\right] n_{x}(\mathbf{r}, t)\right\} \\
+ & \frac{1}{2} \frac{\partial^{2}}{\partial x^{2}}\left\{\left[\phi_{V}(x)-\frac{4}{3} \epsilon \frac{d \phi_{V}(x)}{d x}+\frac{1}{3} \frac{d \phi_{V}^{2}(x)}{d x^{2}}\right] n_{x}(\mathbf{r}, t)\right\} \\
+ & \frac{1}{6} \frac{\partial^{3}}{\partial x^{3}}\left\{\left[\epsilon \phi_{V}(x)-2 \frac{d \phi_{V}(x)}{d x}+\frac{3}{2} \epsilon \frac{d \phi_{V}^{2}(x)}{d x^{2}}\right.\right. \\
- & \left.\left.\frac{1}{4} \frac{d \phi_{V}^{3}(x)}{d x^{3}}\right] n_{x}(\mathbf{r}, t)\right\} \\
+ & \frac{1}{24} \frac{\partial^{4}}{\partial x^{4}}\left\{\left[4 \phi_{V}(x)-12 \epsilon \frac{d \phi_{V}(x)}{d x}+3 \frac{d \phi_{V}^{2}(x)}{d x^{2}}\right.\right. \\
- & \left.\left.\frac{8}{5} \epsilon \frac{d \phi_{V}^{3}(x)}{d x^{3}}+\frac{1}{5} \frac{d \phi_{V}^{4}(x)}{d x^{4}}\right] n_{x}(\mathbf{r}, t)\right\} \\
+ & \tilde{S}(x) .
\end{aligned}
$$

The third and fourth order terms correspond, respectively, to the terms with third and fourth derivates of the expressions in curly braces. Explicit expressions are provided in the Appendix for the moments $\left\langle Q^{3}\right\rangle$ and $\left\langle Q^{4}\right\rangle$ from which the higher order terms above are derived.

\subsection{Uniform expansion}

Eqs. (34) and (35) continue to apply in an isotropic and homogeneous expanding medium if $n_{x}$ is replaced by $\tilde{n}_{x}=$ $\left(R / R_{0}\right)^{2} n_{x}$ and $D \tilde{n}_{x} / D \tau$ is given by

$\frac{D \tilde{n}_{x}(\tau)}{D \tau}=\frac{\partial \tilde{n}_{x}(\tau)}{\partial \tau}-\gamma \frac{\partial \tilde{n}_{x}(\tau)}{\partial x}$,

where

$$
\gamma=\frac{\nu_{0} H}{\chi_{0} c}=\frac{8 \pi}{3} \frac{H}{\lambda_{0}^{3} n_{l} A_{u l}}
$$

is the ratio of the scattering time at line-centre to the expansion time $H^{-1}$ and is known as the Sobolev parameter (Chugai 1980; Rybicki \& Dell'Antonio 1994). It is also the inverse of the optical depth at line-centre $\lambda_{0}$ in a homogeneous and isotropic expanding universe with Hubble parameter $H=\dot{R} / R$ (Field 1959a).

\subsection{Method of solution}

It is instructive to express Eq. (35) in Fourier space. Denoting the Fourier transform of $n_{x}(\mathbf{r}, \tau)$ by $\hat{n}_{\kappa}(\mathbf{r}, \tau)$, Eq. (35) becomes

$$
\begin{aligned}
& \frac{D \hat{n}_{\kappa}(\mathbf{r}, \tau)}{D \tau}- \frac{D \log \omega}{D \tau} \hat{n}_{\kappa}(\mathbf{r}, \tau)= \\
& \frac{\kappa}{2 \pi} \int_{-\infty}^{\infty} d \alpha\left\{\left[\frac{1}{2} \alpha+\frac{1}{6} \kappa\left(\alpha^{2}-3\right)\right.\right. \\
&\left.+\frac{1}{24} \kappa^{2} \alpha\left(\alpha^{2}-8\right)+\frac{1}{24} \kappa^{3}\left(4-3 \alpha^{2}+\frac{1}{5} \alpha^{4}\right)\right] \\
&-i \epsilon\left[1+\frac{2}{3} \kappa \alpha+\frac{1}{4} \kappa^{2}\left(\alpha^{2}-\frac{8}{3}\right)\right. \\
&\left.\left.\quad+\frac{1}{2} \kappa^{3} \alpha\left(\frac{2}{15} \alpha^{2}-1\right)\right]\right\} \hat{\phi}_{V}(\alpha) \hat{n}_{\kappa-\alpha}(\mathbf{r}, \tau),
\end{aligned}
$$

where $\hat{\phi}_{V}(\alpha)$ is the Fourier Transform of $\phi_{V}(x)$. Each order of $\kappa$ corresponds to the same order in the Taylor series expansion. The difference between the second order form above for the non-recoil terms and the Rybicki (2006) form is that in the latter $\alpha / 2$ above is replaced by $\alpha / 2-\alpha^{3} / 6$.

The equation is solved by fourth-order Runge-Kutta with an adaptive timestep. The scheme is not fully stable for terms beyond the third order. While adequate for producing the solutions presented in this paper, it is not sufficiently efficient to integrate to much longer times. Ultimately a modified Crank-Nicholson method would be most desirable for solving Eq. (35). Such an approach is under investigation.

The heating rate expressed in Fourier space may be derived from Eq. (12) and noting that $\hat{\phi}_{V}(-\kappa)=\hat{\phi}_{V}(\kappa)$,

$$
\begin{aligned}
G(\mathbf{r}, t) & =\frac{\chi_{0} c h}{2 \pi} \int_{-\infty}^{\infty} d \kappa\left(\epsilon-\frac{1}{2} i \kappa\right) \hat{\phi}_{V}(\kappa) \hat{n}_{\kappa}(\mathbf{r}, \tau) \\
& =\frac{\chi_{0} c h}{2 \pi} \int_{-\infty}^{\infty} d \kappa\left[\epsilon \hat{n}_{\kappa}^{(r)}(\mathbf{r}, \tau)+\frac{1}{2} \kappa \hat{n}_{\kappa}^{(i)}(\mathbf{r}, \tau)\right] \hat{\phi}_{V}(\kappa)
\end{aligned}
$$

where $\hat{n}_{\kappa}^{(r)}$ and $\hat{n}_{\kappa}^{(i)}$ are the real and imaginary parts of $\hat{n}_{\kappa}$, noting that the real and imaginary parts are, respectively, even and odd functions of $\kappa$ since $n_{x}$ is real. The heating rate may also be evaluated using Eq. (14). To the accuracy of approximation in the treatment here, $\left\langle T_{u}\right\rangle_{H}$ may be replaced by $\left\langle T_{n}\right\rangle_{H}$. Expressed as a ratio of integrals in Fourier space,

$\left\langle T_{n}\right\rangle_{H}=-\frac{h \omega}{k} \frac{\int_{-\infty}^{\infty} d \kappa \hat{n}_{\kappa}^{(r)} \hat{\phi}_{V}(\kappa)}{\int_{-\infty}^{\infty} d \kappa \hat{n}_{\kappa}^{(i)} \kappa \hat{\phi}_{V}(\kappa)}$.

Cosmological expansion is accounted for by identifying $D \hat{n}_{\kappa} / D \tau$ with

$\frac{D \hat{n}_{\kappa}(\tau)}{D \tau}=\frac{\partial \hat{n}_{\kappa}(\tau)}{\partial \tau}+i \gamma \kappa \hat{n}_{\kappa}(\tau)$. 

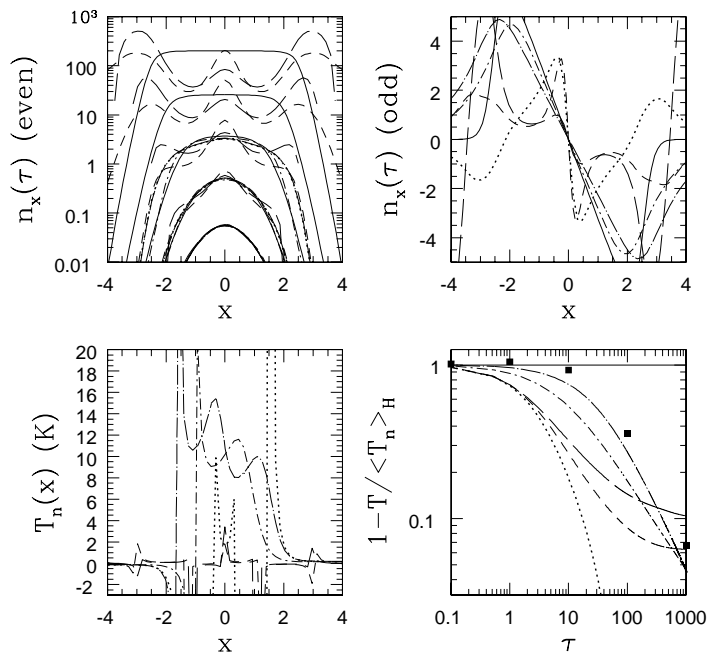

Figure 1. Numerical solution of $n_{x}$ vs $x$ to the Field (1959b) problem for a unit source with a Doppler profile. Shown are the analytic solution (solid), the FPA solutions to second order (shortdashed) and third (long-dashed) order, and the FPDA solutions of Rybicki \& Dell'Antonio (1994) (dotted short-dashed) and Rybicki (2006) (dotted long-dashed). Also shown is a truncated second order FPA solution (dotted line; see text). The effect of atomic recoil is included for a medium at $T=10 \mathrm{~K}$. Top left panel: The even part of $n_{x}$ at the times, from bottom to top, $\tau=0.1,1,10$, 100 and 1000. Only the second and third order FPA solutions are shown at $\tau=100$ and 1000 for clarity. In the absence of recoils, the even part is identical to the full solution. Top right panel: The odd part of $n_{x}$. The odd part vanishes in the absence of atomic recoil. Bottom left panel: The specific temperature $T_{n}(x)$ at $\tau=1000$. Bottom right panel: The evolution of the mean harmonic temperature $\left\langle T_{n}\right\rangle_{H}$ in terms of the heating efficiency. The points correspond to the approximation Eq. (19) applied to the Rybicki (2006) solution.

It is noted that even for an even source in $x$, cosmological expansion will introduce an imaginary part to $\hat{n}_{\kappa}$. It follows from Eq. (39) that energy exchange will occur even without atomic recoil. This results in a cooling term as the photon distribution is redshifted through the line-profile (cf, Chen \& Miralda-Escudé 2004). It is also noted that for a linear inflow of matter, $\gamma$ changes sign and produces a heating term as photons are blueshifted through the line-profile.

\section{RESULTS}

Field (1959b) derived an analytic solution to the problem of the scattering of resonant photons in a homogeneous and isotropic medium with a Doppler profile source term. The problem is not particularly well-suited to the FokkerPlanck approximation because of the rapidly varying source function, but it does provide a test of the approximation and its convergence. Solutions to Eq. (35) are found with $S(x)=\exp \left(-x^{2}\right) / \pi^{1 / 2}$ for four cases: the second and third order FPA equations, and the FPDA equations of Rybicki
\& Dell'Antonio (1994) and Rybicki (2006). The results for cold hydrogen gas at $T=10 \mathrm{~K}$ are shown in Figure 1 . The solutions are separated into their even and odd parts, as, for a source even in $x$, the effect of atomic recoil appears only in the odd part of the solution, which is proportional to $\epsilon$. (The contribution of recoils to the even part is of or$\operatorname{der} \epsilon^{2}$.) The original Rybicki \& Dell'Antonio approximation best matches the analytic solution near the line-centre. The solution of Rybicki (2006) agrees well, but not quite as well as the original Rybicki \& Dell'Antonio (1994) form.

By contrast, the second order Fokker-Planck approximation derived here shows appreciable features. The subtraction of $\alpha^{3} / 6$ from $\alpha / 2$ in Eq. (38) in the Rybicki (2006) formulation plays the critical role of eliminating the oscillatory behaviour. This corresponds to the highest (fourth order) derivative of $\phi_{V}(x)$ in Eq. (34). Truncating the equation at the third derivative of $\phi_{V}(x)$ by subtracting this term from the second order solution eliminates the oscillatory behaviour. Accordingly, a truncated second order FPA (TFPA) equation is solved with this term removed. The term, however, is removed only from the equation for the real part of $\hat{n}_{\kappa}$, not the imaginary, for the reason explained below. Although the (full) third order Fokker-Planck solution agrees more closely with the analytic solution than the second order, the oscillatory behaviour is still present.

Field (1959b) demonstrated that when atomic recoil is included, the radiation field reaches statistical equilibrium with the matter near the line-centre, in the sense that for $\epsilon|x|<<1$, the radiation field asymptotically approaches the form $n_{x} \sim n_{x}^{(e)}[1-2 \epsilon x] \simeq n_{x}^{(e)} \exp [-h(\nu-\nu) / k T]$ for $\tau>>1$. It follows from Eq. (21) that the colour temperature at linecentre is $T_{n}(0)=T$. Since the analytic correction to the profile due to recoil is proportional to $x$, the extent over which the profile assumes this form is unclear. Field (1959b) argued it should apply within a flattened core with the timeasymptotic value $n_{x}(\tau) \simeq \tau /\left[2(\log \tau)^{1 / 2}\right]$ at line-centre, at frequencies smaller than a critical frequency $x_{c}=(\log \tau)^{1 / 2}$. In Figure 1, the odd part of the analytic solution is plotted as $n_{x}^{(o)}=n_{x}^{(e)}[\exp (-2 \epsilon x)-\exp (2 \epsilon x)] / 2$, noting that it is strictly applicable only for $\epsilon|x|<<1$. The slow logarithmic growth of the critical frequency suggests the radiation field may reach statistical equilibrium outside the Doppler core only very slowly. Moreover, the value of the colour temperature depends also on how flat the core is away from line-centre. This suggests the heating rate may not become vanishingly small for many scattering times at line-centre $(\tau>>1)$.

Figure 1 shows that the frequency-specific colour temperature $T_{n}(\nu)$ approaches the gas temperature at linecentre only for the Rybicki \& Dell'Antonio (1994) and Rybicki (2006) approximations. Even for these cases, $T_{n}(\nu)$ deviates considerably from the gas temperature well within the core of the line profile. In the other cases, the colour temperature takes on mostly very small values, both positive and negative. The lesson would appear to be that this temperature has little physical meaning, except possibly very near the line-centre. The colour temperature in the FokkerPlanck approximation, however, appears to converge to the equilibrium temperature at line-centre only very slowly with increasing order. Much away from line-centre, it is not even clear the colour temperature should converge to the gas temperature, except for the extreme blackbody limit.

As discussed in the previous section, both the heat- 
ing rate and the Wouthuysen-Field efficiency depend on the mean harmonic temperature $\left\langle T_{n}\right\rangle_{H}$. Weighting by the line profile produces a mean harmonic temperature that evolves toward the matter temperature for large $\tau$, as shown in Figure 1.

It is noted that while subtracting $\alpha^{3} / 6$ from $\alpha / 2$ in the equation for the real part of $\hat{n}_{\kappa}$ in Eq. (38) removes the oscillations in the second order FPA solution, subtracting this term from the equation for the imaginary part as well produces a solution that results in a rapid, and unphysical, decline in $\left\langle T_{n}\right\rangle_{H}$ to values well below the gas temperature. Subtracting the term only from the real part yields $\left\langle T_{n}\right\rangle_{H} \rightarrow 9.8 \mathrm{~K} \simeq T$ by $\tau \gtrsim 100$, and stays constant at this value up to $\tau=1000$. The light temperature has reasonably converged to the matter temperature within the error of the approximation. The truncated version of the second order FPA equation thus appears a viable approximation for including the effects of atomic recoil while recovering the correct solution to the radiative transfer equation, in spite of the lack of a mathematical justification.

The form of $T_{n}(\nu)$ in the different approximations has a curious property. While it agrees nearly exactly with the gas temperature in the Rybicki \& Dell'Antonio (1994) and Rybicki (2006) Fokker-Planck diffusion approximations at line centre, this is no longer true in the full Fokker-Planck approximations. In these cases, the cancellations among the negative and positive values of $T_{n}(\nu)$ ensure the mean harmonic average colour temperature $\left\langle T_{n}\right\rangle_{H}$ converges to the gas temperature. For the Rybicki \& Dell'Antonio (1994), Rybicki (2006) and truncated second order Fokker-Planck approximations, the convergence is rapid, with the efficiency factor scaling roughly like $1-T /\left\langle T_{n}\right\rangle_{H} \sim \tau^{-1 / 2}$ for $\tau>>1$. For the other cases, the convergence starts rapidly, but by $\tau>100$ levels off at efficiency values of $5-10 \%$ by $\tau=1000$. It is unclear how large $\tau$ must be before the efficiency declines to $1 \%$ and heating essentially shuts off. Going to third order indeed suggests the time is longer than given by the second order Fokker-Planck approximation. The integration method used is too inefficient to integrate to much longer times or to higher orders, so that it is not possible to answer this question here.

The points in the lower right hand panel of Figure 1 correspond to Eq. (19) applied to the Rybicki (2006) solution. At early times this results in efficiency factors exceeding unity, which is unphysical. At late times, the approximation agrees reasonably well with the Rybicki (2006) predicted efficiency.

The case of a constant continuum source $S(x)=1$ is shown in Figure 2. The gas temperature is $T=10 \mathrm{~K}$, corresponding to $a=0.015$ for the Voigt profile. The second and third order FPA solutions again show substantial structure, and the efficiencies reach plateaus for $\tau>100$ at values of $15-20 \%$ by $\tau=1000$. Again, heating persists at a nonnegligible level for many scattering times at line centre. For the two FPDA and the TFPA solutions, the heating efficiency continues to decline, again scaling roughly as $\tau^{-1 / 2}$ for $\tau>>1$. The points in the right hand panel correspond to the approximation Eq. (19) for the efficiency applied to the Rybicki (2006) solution. The values are again unphysically high at early times.
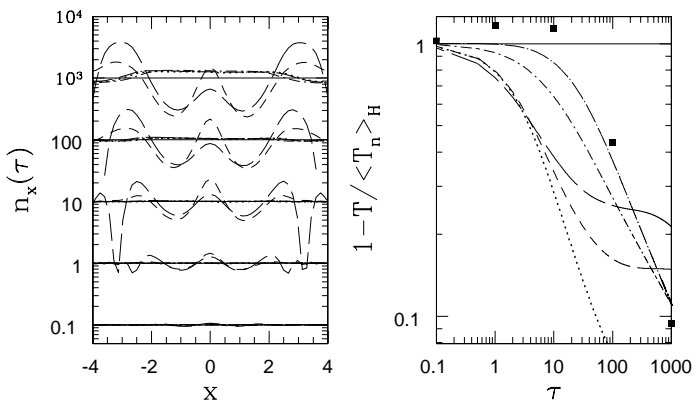

Figure 2. Numerical solution of $n_{x}$ vs $x$ for a constant continuum source. A Voigt scattering profile is adopted with a gas temperature $T=10 \mathrm{~K}$, corresponding to $a=0.015$. Shown are the no-scattering solution (solid), the FPA solutions to second order (short-dashed) and third order (long-dashed), and the FPDA solutions of Rybicki \& Dell'Antonio (1994) (dotted short-dashed) and Rybicki (2006) (dotted long-dashed). Also shown is a truncated second order FPA solution (dotted line; see text). The effect of atomic recoil is included for a medium at $T=10 \mathrm{~K}$. Left panel: The evolution of $n_{x}$ at the times, from bottom to top, $\tau=0.1$, 1, 10, 100 and 1000. Right panel: The evolution of the mean harmonic temperature $\left\langle T_{n}\right\rangle_{H}$ in terms of the heating efficiency. The points correspond to the approximation Eq. (19) applied to the Rybicki (2006) solution.

\section{DISCUSSION \& CONCLUSIONS}

\subsection{Fokker-Planck approximations}

Previous formulations of the time-dependent Fokker-Planck approximation to the radiative transfer equation for the scattering of resonant photons were based on a Taylor series expansion of only the radiation intensity in the scattering integral (Unno 1955; Harrington 1973; Rybicki \& Dell'Antonio 1994; Rybicki 2006). The result of the approximation has the form of a diffusion equation. In this paper, an equation based on a Taylor series expansion of the product of the scattering probability and the intensity is obtained, which is the standard Fokker-Planck approximation. This permits an easier analysis of the improved accuracy of the approximation to higher orders. In this paper, the resulting approximation has been derived for Case II scattering using the full Voigt profile, and including the first-order contributions from atomic recoil. The approximation correctly conserves particle numbers with well-defined coefficients, unlike the Fokker-Planck diffusion approximation.

Time-dependent solutions are presented to the FPDA and FPA equations for the case of a unit Doppler profile source (for Case I) and for a constant continuum source. An additional term in the second order FPA equation intro- 
duces artificial features within the cores of the profiles. These diminish in the third order, but are still pronounced. Truncating the second order FPA equation by removing this term eliminates the features. In principle, the features should be eliminated by going to a sufficiently high order in the FokkerPlanck expansion. Such an investigation, however, requires an alternative integration method to that used here.

\subsection{Approach to statistical equilibrium}

An expression for the heating rate of the scattering medium through atomic recoil is derived. It is shown that the FokkerPlanck approximation correctly recovers the full heating rate to the accuracy of the approximation. Both the heating rate and the effectiveness of the Wouthuysen-Field mechanism depend on the mean harmonic colour temperature $\left\langle T_{u}\right\rangle_{H}$ computed from the frequency-specific temperture $T_{u}(\nu)$, weighted by the absorption profile. For photons of frequency $\nu$ in statistical equilibrium with the scattering medium at temperature $T$, it has usually been expected that $T_{u}(\nu)$ converges to $T$ for large $\tau$, where $\tau$ is the time expressed in units of the scattering time at line-centre. This was shown to be the case analytically by Field (1959b) for two particular problems involving Case I redistribution, at least near the line centre. Numerical solutions are found to Field's problem for a pure Doppler profile source. Using the FPDA approximations of Rybicki \& Dell'Antonio (1994) and Rybicki (2006), $T_{u}(\nu) \rightarrow T$ is found at the line centre for $\tau>>1$, but away from the line centre $T_{u}(\nu)$ deviates appreciably from $T$, and even takes on negative values. For the FPA equations, $\left|T_{u}(\nu)\right|$ is generally small compared with $T$, and takes on both negative and positive values. The third order FPA equation predicts the larger value at line centre. Presumably higher order Fokker-Planck approximations must yield the correct temperature at the line-centre. The investigation of this question is in progress.

The more physically-motivated quantity $\left\langle T_{u}\right\rangle_{H}$ is found to converge toward $T$. The rate of convergence, however, is sensitive to the form of the approximation. The most rapid convergence is found for the truncated Fokker-Planck approximation. Whether or not this is an artifact of the form of the approximation is unclear. Convergence is also found for the Fokker-Planck diffusion approximations. For the full Fokker-Planck approximation developed here, the convergence of $\left\langle T_{u}\right\rangle_{H}$ to $T$ substantially slows for $\tau>100$. Going to third order results in an even slower convergence rate.

\subsection{Angular dependence}

The results presented have all assumed an isotropic radiation field. In situations in which the gas is heated by one or more local sources, the incident radiation field may be highly anisotropic. One approach is to take angular moments of the radiative transfer equation. Neglecting stimulated emission, the upper level population in equilibrium is given by

$n_{u}(\nu) A_{u l}=n_{l} \frac{B_{l u}}{4 \pi} \oint \frac{d \omega^{\prime}}{4 \pi} \int d \nu^{\prime} R\left(\nu^{\prime}, \hat{\mathbf{n}}^{\prime} ; \nu, \hat{\mathbf{n}}\right) I_{\nu^{\prime}}\left(\mathbf{r}, t, \hat{\mathbf{n}}^{\prime}\right),($

and the emissivity becomes

$\eta_{\nu}=h \nu n_{l} \frac{B_{l u}}{4 \pi} \oint \frac{d \omega^{\prime}}{4 \pi} \int d \nu^{\prime} R\left(\nu^{\prime}, \hat{\mathbf{n}}^{\prime} ; \nu, \hat{\mathbf{n}}\right) I_{\nu^{\prime}}\left(\mathbf{r}, t, \hat{\mathbf{n}}^{\prime}\right)$.
The angular coupling between the redistribution function and the intensity precludes a simple form for the energy transfer rate. A solution to the radiative transfer equation along these lines is sketched by Rybicki \& Dell'Antonio (1994).

\subsection{Astrophysical implications}

The Wouthuysen-Field mechanism for coupling the spin temperature to the colour temperature of resonant photons has been discussed in a variety of contexts, including the IGM, intergalactic gas clouds like Damped Lyman-Alpha Absorbers, gas on the peripheries of galaxies, and in clouds near quasars, with applications not only to ${ }^{1} \mathrm{H}$, but ${ }^{2} \mathrm{D}$ and ${ }^{3} \mathrm{He}^{+}$as well (eg, Field 1958; Deguchi \& Watson 1985). The mechanism may be particularly complicated for ${ }^{3} \mathrm{He}^{+}$because of the Ly $\alpha$ radiation from ${ }^{4} \mathrm{He}^{+}$and the coincidence of the ${ }^{3} \mathrm{He}^{+}$Ly $\alpha$ transition with a Bowen fluorescence line of O III . The reader is referred to Deguchi \& Watson (1985) for a discussion of these and other complicating effects. The discussion in this section is confined to more general remarks.

The scattering times at line-centre for ${ }^{1} \mathrm{H},{ }^{2} \mathrm{D}$ and ${ }^{3} \mathrm{He}^{+}$, normalised to the hydrogen number density $n_{\mathrm{H}}\left(\right.$ in $\left.\mathrm{cm}^{-3}\right)$ using the Big Bang Nucleosynthesis constrained abundances of ${ }^{2} \mathrm{D}$ and ${ }^{3} \mathrm{He}$ from Burles, Nollett \& Turner (2001), are

$$
\begin{aligned}
t_{\mathrm{sca}} & =3.2 n_{\mathrm{H}}^{-1} T^{1 / 2} \mathrm{~s} \quad ;{ }^{1} \mathrm{H}, \\
& =7.3 \times 10^{4} n_{\mathrm{H}}^{-1} T^{1 / 2} \mathrm{~s} \quad ;{ }^{2} \mathrm{H}, \\
& =1.0 \times 10^{4} n_{\mathrm{H}}^{-1} T^{1 / 2} \mathrm{~s} \quad ;{ }^{3} \mathrm{He}^{+} .
\end{aligned}
$$

These timescales are typically short for neutral gas. For ionized gas, however, as for instance in Lyman Limit Systems, the rates may become quite long, particularly for ${ }^{2} \mathrm{D}$ and ${ }^{3} \mathrm{He}^{+}$. For instance, in a cloud with $n_{\mathrm{HI}} \approx 10^{-5} \mathrm{~cm}^{-3}$ at $T=10^{4} \mathrm{~K}$, the timescale is about $10^{12} \mathrm{~s}$ for these isotopes. If it takes 10-100 scattering times for the Wouthuysen-Field mechanism to bring the colour temperature $\left\langle T_{u}\right\rangle_{H}$ to the gas temperature, the equilibrium timescale approaches that of the lifetime of massive stars. If such objects, or their $\mathrm{H}$ II regions, are the sources of the Ly $\alpha$ photons, it may be that while the colour temperature at the $\mathrm{H}$ i resonance has come into equilibrium, that of $\mathrm{D}$ I has not, in which case an erroneous $\mathrm{D} / \mathrm{H}$ ratio may be inferred from the comparison of radio measurements of the two hyperfine transitions. Conversely, if the cosmic $\mathrm{D} / \mathrm{H}$ ratio is accepted, such observations may provide an estimate of the time since the radiation sources turned on. Even for hydrogen, the colour temperature may not reach equilibrium if the source varies on a timescale that does not greatly exceed the scattering time.

Cosmological expansion will generally play a negligible role in the approach to statistical equilibrium of the radiation field. This is because the smallness of the Sobolev parameter. Rybicki \& Dell'Antonio (1994) show that, within the damping wings, expansion negligibly affects the photon distribution until a time $\tau \approx\left(a / \gamma^{4}\right)^{1 / 3}>>1$ for typical values of $a$ and $\gamma$ expected for the IGM.

Expansion, however, may play a role in more local situations, such as the outflow from a star or an active galactic nucleus (AGN). One possible effect is a freeze-out of $\left\langle T_{u}\right\rangle_{H}$. For the sake of demonstrating the principle, a computation with $a=0.015$ and $\gamma=0.1$ is show in Figure 3 for cold hy- 

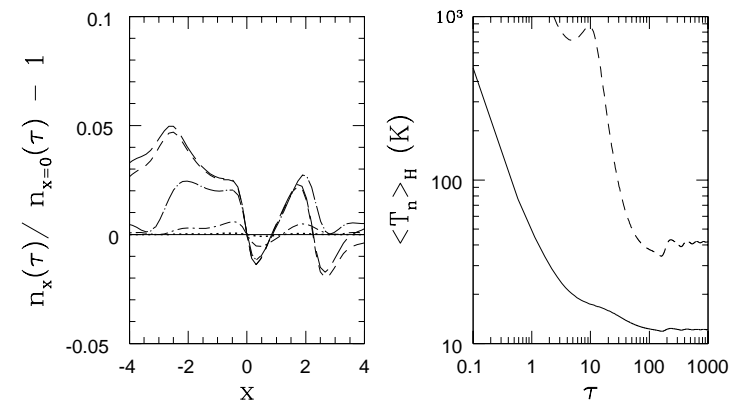

Figure 3. Numerical solution of $n_{x}$ vs $x$ for a constant continuum source in an expanding medium with $\gamma=0.1$ A Voigt scattering profile is adopted with a gas temperature $T=10 \mathrm{~K}$, corresponding to $a=0.015$. The results for the truncated FPA solution are shown in the left panel at the times $\tau=0.1$ (dotted), 1 (dot short-dashed), 10 (dot long-dashed), 100 (short-dashed) and 1000 (long-dashed). The photons are increasingly redshifted with time. The light temperature $\left\langle T_{n}\right\rangle_{H}$ is shown in the right panel for the truncated second order FPA equation (solid line) and the Rybicki (2006) FPDA equation (dashed line).

drogen gas at $T=10 \mathrm{~K}$. The photon distribution is skewed to the red by the expansion. Both the truncated second order FPA solution and the Rybicki (2006) FPDA solution predict a freeze-out of $\left\langle T_{u}\right\rangle_{H}$ above the gas temperature for $\tau>100$, but at different values. The radiation density profiles differ slightly near line centre, with the dip at $x=0$ in Figure 3 not as deep in the FPDA solution.

For hot ionised hydrogen gas, the expansion may result in a light temperature well below the matter temperature. This is illustrated in Figure 4 for $a=3.3 \times 10^{-4}$ and $\gamma=0.01$ for gas at $T=2 \times 10^{4} \mathrm{~K}$. The photon distribution is strongly skewed toward the red by $\tau=1000$, with a sharp cut-off in the blue similar to the Chugai (1980) steady-state limiting solution for the radiative transfer in the profile wings of photons from a monochromatic source at line-centre in an expanding medium. Both the truncated second order FPA solution and the Rybicki (2006) FPDA solution predict $\left\langle T_{u}\right\rangle_{H}<T$ for $\tau \gtrsim 60$. The Rybicki (2006) FPDA predicts negative values of $\left\langle T_{u}\right\rangle_{H}$ for $\tau \lesssim 50$. It should be noted, however, that at the assumed temperature the requirement $2 \epsilon<<b / c$ is only marginally met. The full Rybicki (2006) formulation includes an extra term of order $b / c$ which is not incorporated into the formalism here. This term may eliminate the negative values, although it is noted that negative values are still found in a similar integration using $T=1000 \mathrm{~K}$.

The possible heating role played by atomic recoil was
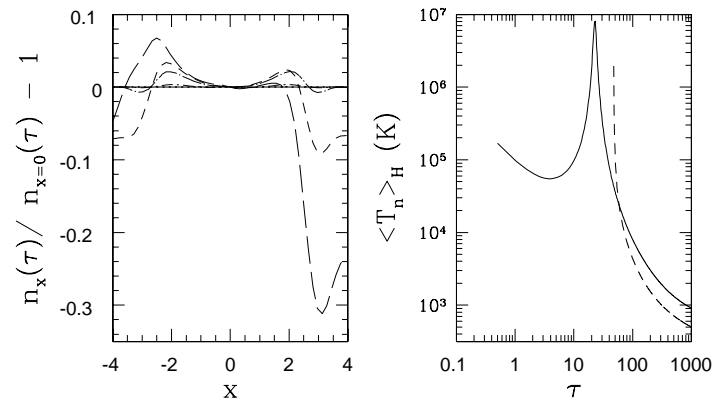

Figure 4. Numerical solution of $n_{x}$ vs $x$ for a constant continuum source in an expanding medium with $\gamma=0.01$. A Voigt scattering profile is adopted with a gas temperature $T=2 \times 10^{4} \mathrm{~K}$, corresponding to $a=3.3 \times 10^{-4}$. The results for the truncated FPA solution are shown in the left panel at the times $\tau=0.1$ (dotted), 1 (dot short-dashed), 10 (dot long-dashed), 100 (short-dashed) and 1000 (long-dashed). The photons are increasingly redshifted with time. The light temperature $\left\langle T_{n}\right\rangle_{H}$ is shown in the right panel for the truncated second order FPA equation (solid line) and the Rybicki (2006) FPDA equation (dashed line).

originally proposed by MMR as a possible means of heating the IGM at high redshifts before it is re-ionised. Given the rapidity with which $\left\langle T_{u}\right\rangle_{H} \rightarrow T$, this now appears unlikely (cf, Chen \& Miralda-Escudé 2004; Rybicki 2006), particularly if the predictions using the Rybicki \& Dell'Antonio (1994), Rybicki (2006) or truncated second order FokkerPlanck approximations are most accurate. The prediction of the FPA equations, however, is a levelling off of the efficiency to values of $10-20 \%$ for large $\tau$. This suggests that heating may persist for $\tau>>1$. How long is still unclear. The delay may also be an artifact of the artificial oscillatory behaviour of the solutions.

The heating rate of MMR at full efficiency may be expressed as

$G_{\mathrm{MMR}}=\frac{h \nu_{0}}{m_{a} c^{2}} h \nu_{0} n_{l} P_{l}$.

The amount of energy per atom in the lower level that may deposited before the efficiency becomes vanishingly small may be expressed as

$\Delta E=f\left(G_{\mathrm{MMR}} / n_{l}\right) t_{\mathrm{sca}}=f \frac{h \nu_{0}}{m_{a} c^{2}} \frac{\omega \bar{n}_{\nu_{0}}}{n_{l}} h \nu_{0}$,

where $\bar{n}_{\nu_{0}}=\int d \nu \phi_{V}(\nu) n_{\nu}$. For the hydrogen Ly $\alpha$ transition, $h \nu_{0} / m_{a} c^{2} \approx 10^{-8}$ and $h \nu_{0} \gtrsim 10^{5} \mathrm{~K}$. Consequently a very large ratio of photons within a Doppler width of the resonance to neutral hydrogen atoms is typically required for an appreciable increase in temperature. Expressed 
in terms of the average photon occupation number $\overline{\mathcal{N}}\left(\nu_{0}\right)$ at line-centre,

$$
\begin{aligned}
\Delta E & =f \frac{h \nu_{0}}{m_{a} c^{2}} \frac{b}{c} \frac{8 \pi \overline{\mathcal{N}}\left(\nu_{0}\right)}{n_{l} \lambda_{0}^{3}} h \nu_{0} \\
& \approx 8 \times 10^{-14}\left(\frac{f}{10}\right) T^{1 / 2} n_{l}^{-1}\left[\frac{\overline{\mathcal{N}}\left(\nu_{0}\right)}{10^{-21}}\right] \mathrm{K},
\end{aligned}
$$

where the expression has been evaluated for hydrogen Ly $\alpha$ in the last line, and normalised to $\overline{\mathcal{N}}\left(\nu_{0}\right)=10^{-21}$, corresponding to a mean intensity of $\bar{J}_{\nu_{0}}=2.2 \times$ $10^{-22} \mathrm{erg} \mathrm{cm}^{-2} \mathrm{~s}^{-1} \mathrm{~Hz}^{-1} \mathrm{sr}^{-1}$. Thus in a normal intergalactic environment, the effect is completely negligible even prior to the reionization epoch.

There may be some circumstances, however, where the effect may contribute non-negligibly to the total heating. For instance, for blackbody radiation at $T=10^{4} \mathrm{~K}, \overline{\mathcal{N}}\left(\nu_{0}\right) \approx$ $\exp \left[-h \nu_{0} / k T\right] \approx 8 \times 10^{-6}$. For a somewhat dilute blackbody not in thermal equilibrium with the matter, recoils may provide a significant amount of heating. Whether or not it would be competitive with other processes depends on the particular situation.

At the opposite extreme, cold tenuous gas near a luminous source such as a young star or active galactic nucleus, if shielded from ionizing radiation, could be heated by several degrees or more.

Another situation in which recoil heating may play a role is in an $\mathrm{H}$ in region. According to the on-the-spot approximation, the photon occupation number at line centre due to diffuse recombination radiation is (Osterbrock 1974),

$\mathcal{N}_{d}\left(\nu_{0}\right)=\left(\frac{h^{2}}{2 \pi m_{e} k T}\right)^{3 / 2} \frac{n_{\mathrm{HII}} n_{e}}{n_{\mathrm{HI}}} \approx 4 \times 10^{-22} T_{4}^{-3 / 2} \frac{1}{\chi_{\mathrm{HI}}^{2}} n_{\mathrm{HI}}$,

where $T_{4}$ is $T$ in units of $10^{4} \mathrm{~K}, \chi_{\mathrm{HI}}$ is the neutral hydrogen fraction, and $m_{e}$ is the mass of an electron. Using this in Eq. (47) gives

$\Delta E \approx 3 \times 10^{-12}\left(\frac{f}{10}\right) T_{4}^{-1} \frac{1}{\chi_{\mathrm{HI}}^{2}} \mathrm{~K}$.

It is useful to express this in terms of a source of ionising photons $S$ for a Ly $\alpha$ optical depth $\tau_{\mathrm{HI}}$ through a region of size $R$. Then

$\Delta E \approx 3 S_{50} \tau_{\mathrm{HI}}{ }^{-1} R_{\mathrm{pc}}{ }^{-1} T_{4}^{-1} \mathrm{~K}$,

where $S_{50}$ is in units of $10^{50} \mathrm{~s}^{-1}$ and $R_{\mathrm{pc}}$ is in units of parsecs. The scattering of diffuse Ly $\alpha$ photons may provide a significant source of heat in the development of an $\mathrm{H}$ II region near an ultraluminous star or an AGN.

\section{ACKNOWLEDGMENTS}

The author thanks Peter Brand for helpful conversations.

\section{REFERENCES}

Basko M. M., 1978, Sov. Phys. JETP, 48, 644

Basko M. M., 1981, Astrophysics, 17, 69

Bowman J. D., Morales M. F., Hewitt J. N., 2006, ApJ, 638, 20

Burles S., Nollett K. M., Turner M. S., 2001, ApJ, 552, L1

Chen X., Miralda-Escudé J., 2004, ApJ, 602, 1
Chugai N. N., 1980, Sov. Astron. Lett., 6, 91

Deguchi S., Watson W. D., 1985, ApJ, 290, 578

Field G. B., 1958, Proc. I.R.E., 46, 240

Field G. B., 1959a, ApJ, 129, 536

Field G. B., 1959b, ApJ, 129, 551

Harrington J. P., 1973, MNRAS, 162, 43

Hummer D. G., 1962, MNRAS, 125, 21

Krolik J. H., 1990, ApJ, 353, 21

Madau, P., Meiksin, A., Rees, M. J., 1997, ApJ, 475, 429 (MMR)

Mihalas D., 1978, Stellar Atmospheres, 2nd edn. W. H. Freeman and Co., San Francisco

Milkey R. W., Mihalas D., 1973, ApJ, 185, 709

Osterbrock D. E., 1974, Astrophysics of Gaseous Nebulae. W. H. Freeman and Co., San Francisco

Peebles P. J. E., 1968, ApJ, 153, 1

Peebles P. J. E., 1969, ApJ, 157, 45

Peebles P. J. E., 1993, Principles of Physical Cosmology. Princeton Univ. Press, Princeton

Rybicki G. B., 2006 (astro-ph/0603047)

Rybicki G. B., Dell'Antonio I. P., 1994, ApJ, 427, 603

Tozzi P., Madau P., Meiksin A., Rees M. J., 2000, ApJ, 528, 597

Unno W., 1955, PASJ, 7, 81

Wouthuysen S. A., 1952, AJ, 57, 31

\section{APPENDIX A: MOMENTS OF THE REDISTRIBUTION FUNCTION}

The moments $\langle Q\rangle \varphi(\nu)$ of the redistribution probability $W(\nu, Q)$ are computed here. These results generalize those of Rybicki \& Dell'Antonio (1994) to explicitly take into account atomic recoil. Accordingly, the results are derived here from first principles.

Consider the absorption of a photon of frequency $\nu$ travelling in direction $\hat{\mathbf{n}}$ and re-emission of a photon of frequency $\nu^{\prime}$ travelling in direction $\hat{\mathbf{n}}^{\prime}$ by an atom travelling with velocity $\mathbf{v}$ in the laboratory frame. It is useful to project $\mathbf{v}$ onto an orthogonal basis given by the average and difference of the photons directions (Hummer 1962):

$\hat{\mathbf{n}}_{\mathbf{1}}=\gamma_{+}\left(\hat{\mathbf{n}}+\hat{\mathbf{n}}^{\prime}\right), \quad \hat{\mathbf{n}}_{\mathbf{2}}=\gamma_{-}\left(\hat{\mathbf{n}}-\hat{\mathbf{n}}^{\prime}\right), \quad \hat{\mathbf{n}}_{\mathbf{3}}=\hat{\mathbf{n}} \times \hat{\mathbf{n}}^{\prime},(\mathrm{A} 1)$ where $\gamma_{ \pm}=1 /[2(1 \pm \mu)]^{1 / 2}$ and $\mu=\hat{\mathbf{n}} \cdot \hat{\mathbf{n}}^{\prime}$. The corresponding components of the atom velocity are $\left(v_{1}, v_{2}, v_{3}\right)$. To lowest order in $v / c$ and $h \nu_{0} / m_{a} c^{2}$, where $m_{a}$ is the mass of the recoiling atom, the frequency shift on scattering is given by

$\nu^{\prime}-\nu=\nu_{0}\left[\frac{\mathbf{v}}{c} \cdot\left(\hat{\mathbf{n}}^{\prime}-\hat{\mathbf{n}}\right)-\frac{h \nu_{0}}{m_{a} c^{2}}(1-\mu)\right]$.

Allowing for radiation damping in the upper level, the absorption profile is given by the Lorentz profile in the restframe of the atom

$f(\nu)=\frac{\delta / \pi}{\left[\left(\nu-\nu_{0}\right)^{2}+\delta^{2}\right]}$,

where $\nu_{0}$ is the line-centre frequency and $\delta=\Gamma / 4 \pi$, where $\Gamma$ is the radiative damping width of the upper state. In expression Eq. (A3), the implicit approximation $\nu / \nu_{0} \approx 1$ is made, except for the frequency shift in the denominator, and so does not recover the correct behaviour far from line centre, such as the Rayleigh limit (Peebles 1969; Peebles 1993). Since this paper is concerned solely with energy transfer resulting from resonant scattering, this is an adequate approximation. Radiation heat exchange at other frequencies must be dealt with separately. 
It is assumed that the atomic velocites are distributed as a Maxwellian with temperature $T$ according to

$P(u) d^{3} u=\pi^{-3 / 2} \exp \left(-u^{2}\right) d^{3} u$,

where $\mathbf{u}=\mathbf{v} / b$, and $b=\left(2 k T / m_{a}\right)^{1 / 2}$ is the Doppler parameter. It is useful to introduce the Doppler width $\omega=$ $\nu_{0} b / c$, frequency offset $x=\left(\nu-\nu_{0}\right) / \omega$, recoil parameter $\epsilon=\left(h \nu_{0} / m_{a} c^{2}\right) c / b=h \nu_{0} /\left(2 k T m_{a} c^{2}\right)^{1 / 2}$, and normalised damping constant $a=\delta / \omega$. The frequency shift may then be expressed as $x-x^{\prime}=\gamma_{-}^{-1} u_{2}+\epsilon(1-\mu)$. Then the probability that an incident photon of frequency shift $x$ is scattered to $x^{\prime}=x-q$ is given by

$$
\begin{aligned}
w(x, q, \mu)= & \frac{a}{\pi^{2}} \int_{-\infty}^{\infty} d u_{1} \int_{-\infty}^{\infty} d u_{2} \exp \left(-u_{1}^{2}-u_{2}^{2}\right) \\
& \times \frac{\delta\left[q-\gamma_{-}^{-1} u_{2}-\epsilon(1-\mu)\right]}{a^{2}+\left[x-\gamma_{+} u_{1}(1+\mu)-\gamma_{-} u_{2}(1-\mu)\right]^{2}},
\end{aligned}
$$

neglecting terms of order $x b / c$ in the denominator.

It is noted that the form of $w(x, q, \mu)$ satisfies the asymmetry relation Eq. (8) in the limit $h \nu_{0}>>k T$ and $\nu^{\prime} / \nu \approx 1$ (to order $x b / c$ ). The reverse scattering of $x \rightarrow x^{\prime}=x-q$ is $x^{\prime}=x-q \rightarrow x^{\prime}-(-q)$. The corresponding relation between $w(x, q, \mu)$ and $w(x-q,-q, \mu)$. Expressing $w(x-q,-q, \mu)$ according to Eq. (A5) and making the change of variable $u_{2}=\tilde{u}_{2}-2 \gamma_{-} q$ shows after straightforward manipulations that

$$
\begin{aligned}
w(x-q,-q, \mu) & =e^{-2 \epsilon q} w(x, q, \mu) \\
& =\exp \left[-\frac{h\left(\nu-\nu^{\prime}\right)}{k T}\right] w(x, q, \mu) .
\end{aligned}
$$

The angle-averaged coefficients $\left\langle Q^{n}\right\rangle \varphi(\nu)$ may be expressed in terms of $w(x, q, \mu)$ according to

$\left\langle Q^{n}\right\rangle \varphi(\nu)=\frac{1}{2} \omega^{n-1} \int_{-1}^{1} d \mu a_{n}(x, \mu)$,

where

$a_{n}(x, \mu)=\int_{-\infty}^{\infty} d q q^{n} w(x, q, \mu)$.

Following Rybicki \& Dell'Antonio (1994), such integrals are most easily evaluated in Fourier space. From Eq. (A5), the Fourier transform of $w(x, q, \mu)$ is

$$
\begin{aligned}
\hat{w}(\kappa, \lambda, \mu)= & \int d x \int d q \exp (i x \kappa) \exp (i q \lambda) \\
& \frac{a}{\pi^{2}} \int_{-\infty}^{\infty} d u_{1} \int_{-\infty}^{\infty} d u_{2} \exp \left(-u_{1}^{2}-u_{2}^{2}\right) \\
& \times \frac{\delta\left[q-\gamma_{-}^{-1} u_{2}-\epsilon(1-\mu)\right]}{a^{2}+\left[x-\gamma_{+} u_{1}(1+\mu)-\gamma-u_{2}(1-\mu)\right]^{2}} \\
= & \exp \left[-a|\kappa|-\frac{1}{4} \kappa^{2}+i \lambda \epsilon(1-\mu)\right. \\
& \left.-\frac{1}{2} \lambda \kappa(1-\mu)-\frac{1}{2} \lambda^{2}(1-\mu)\right]
\end{aligned}
$$

The Fourier transform of $a_{n}(x, \mu)$ may be expressed as

$\hat{a}_{n}(\kappa, \mu)=\left.\frac{\partial^{n}}{\partial(i \lambda)^{n}} \hat{w}(\kappa, \lambda, \mu)\right|_{\lambda=0}$, noting that factors of $q^{n}$ correspond to $n$ derivatives with respect to $i \lambda$ in Fourier space. The first five values of $\hat{a}_{n}(\kappa, \mu)$ are then

$$
\begin{aligned}
\hat{a}_{0}(\kappa, \mu)= & \exp \left[-a|\kappa|-\frac{1}{4} \kappa^{2}\right] ; \\
\hat{a}_{1}(\kappa, \mu)= & {\left[\epsilon(1-\mu)+\frac{1}{2} i \kappa(1-\mu)\right] } \\
& \times \exp \left[-a|\kappa|-\frac{1}{4} \kappa^{2}\right] ; \\
\hat{a}_{2}(\kappa, \mu)= & {\left[(1-\mu)+(1-\mu)^{2}\left(i \epsilon \kappa-\frac{1}{4} \kappa^{2}\right)\right] } \\
& \times \exp \left[-a|\kappa|-\frac{1}{4} \kappa^{2}\right] ; \\
\hat{a}_{3}(\kappa, \mu)= & {\left[3(1-\mu)^{2}\left(\epsilon+\frac{1}{2} i \kappa\right)\right.} \\
& \times \exp \left[-a|\kappa|-\frac{1}{4} \kappa^{2}\right] ; \\
& {\left[3(1-\mu)^{2}+6(1-\mu)^{3}\left(i \epsilon \kappa-\frac{1}{4} \kappa^{2}\right)\right.} \\
& \left.+\frac{1}{16}(1-\mu)^{4}\left(-8 i \epsilon \kappa^{3}+\kappa^{4}\right)\right] \\
\left.\hat{a}_{4}(\kappa, \mu)\right] & \times \exp \left[-a|\kappa|-\frac{1}{4} \kappa^{2}\right] .
\end{aligned}
$$

The $n=0$ term corresponds to the full absorption profile $\varphi(\nu)$. Since for Case II redistribution, this corresponds to the Voigt profile, the notation $\varphi_{V}(\nu)$ is adopted for $\varphi(\nu)$. Its dimensionless form will be denoted as $\phi_{V}(x)$. The Fourier transform of $\phi_{V}(x)$ is given by $\hat{a}_{0}(\kappa, \mu)$ in Equation (A11). The first five moments of $Q$ are then

$$
\begin{aligned}
\left\langle Q^{0}\right\rangle= & 1 ; \\
\langle Q\rangle= & \epsilon \omega-\frac{1}{2} \omega^{2} \frac{d \log \varphi_{V}(\nu)}{d \nu} ; \\
\left\langle Q^{2}\right\rangle= & \omega^{2}-\frac{4}{3} \epsilon \omega^{3} \frac{d \log \varphi_{V}(\nu)}{d \nu} \\
& +\frac{1}{3} \omega^{4} \frac{1}{\varphi_{V}(\nu)} \frac{d^{2} \varphi_{V}(\nu)}{d \nu^{2}} ; \\
\left\langle Q^{3}\right\rangle= & 4 \epsilon \omega^{3}-2 \omega^{4} \frac{d \log \varphi_{V}(\nu)}{d \nu}+\frac{3}{2} \epsilon \omega^{5} \frac{1}{\varphi_{V}(\nu)} \frac{d^{2} \varphi_{V}(\nu)}{d \nu^{2}} \\
& -\frac{1}{4} \omega^{6} \frac{1}{\varphi_{V}(\nu)} \frac{d^{3} \varphi_{V}(\nu)}{d \nu^{3}} ; \\
\left\langle Q^{4}\right\rangle= & 4 \omega^{4}-12 \epsilon \omega^{5} \frac{d \log \varphi_{V}(\nu)}{d \nu}+3 \omega^{6} \frac{1}{\varphi_{V}(\nu)} \frac{d^{2} \varphi_{V}(\nu)}{d \nu^{2}} \\
& -\frac{8}{5} \epsilon \omega^{7} \frac{1}{\varphi_{V}(\nu)} \frac{d^{3} \varphi_{V}(\nu)}{d \nu^{3}} \\
& +\frac{1}{5} \omega^{8} \frac{1}{\varphi_{V}(\nu)} \frac{d^{4} \varphi_{V}(\nu)}{d \nu^{4}} .
\end{aligned}
$$

